DE

M E D I C I N A

T R O P I C A L

$\mathrm{DE}$

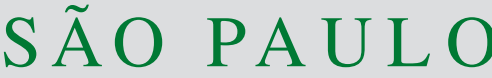

JOURNAL OF THE SÃO PAULO INSTITUTE OF TROPICAL MEDICINE

(1) Centro Universitário Cesumar, Departamento de Biomedicina, Maringá, Paraná, Brazil

(2) Universidade de São Paulo, Faculdade de Medicina, Departamento de Moléstias Infecciosas, São Paulo, São Paulo, Brazil

(3) Instituto de Infectologia Emilio Ribas, São Paulo, São Paulo, Brazil

(4) Universidade de São Paulo, Instituto de Medicina Tropical de São Paulo, Laboratório de Soroepidemiologia e Imunobiologia (LIM 38 HC-FMUSP), São Paulo,

São Paulo, Brazil

Correspondence to: José Angelo Lauletta Lindoso

Universidade de São Paulo, Instituto de Medicina Tropical de São Paulo, Laboratório de Soroepidemiologia e Imunobiologia, Av. Dr. Enéas Carvalho de Aguiar, 470, CEP 05403-000, São Paulo, SP, Brazil Tel: +55 1130617023 .

E-mail: jlindoso@usp.br

Received: 23 September 2016

Accepted: 22 February 2017

\section{Efficiency of noninvasive sampling methods (swab) together with Polymerase Chain Reaction (PCR) for diagnosing American Tegumentary Leishmaniasis}

\author{
Sara Macente Boni ${ }^{1,2}$, Luiza Keiko Oyafuso ${ }^{3}$, Rita de Cassia Soler ${ }^{3}$, \\ José Angelo Lauletta Lindoso $0^{3,4}$
}

\section{ABSTRACT}

Traditional diagnostic methods used to detect American Tegumentary Leishmaniasis, such as histopathology using biopsy samples, culture techniques, and direct search for parasites, have low sensitivity and require invasive collection procedures. This study evaluates the efficiency of noninvasive sampling methods (swab) along with Polymerase Chain Reaction (PCR) for diagnosing American Tegumentary Leishmaniasis using skin and mucous samples from 25 patients who had tested positive for leishmaniasis. The outcome of the tests performance on swab samples was compatible with PCR results on biopsy samples. The findings have also shown that PCR-kDNA test is more efficient than PCR-HSP70 and qPCR tests (sensitivity of $92.3 \%, 40.7 \%$, and $41 \%$, respectively). Given the high sensitivity of the tests and the fact that the sampling method using swabs affords greater patient comfort and safety, it could be said that this method is a promising alternative to conventional biopsybased methods for the molecular diagnosis of leishmaniasis.

KEYWORDS: Tegumentary Leishmaniasis. Polymerase Chain Reaction. Swab. Leishmania braziliensis.

\section{INTRODUCTION}

American Tegumentary Leishmaniasis (ATL) is a disease caused by protozoan parasites of the genus Leishmania and transmitted by the bite of infected sand flies ${ }^{1}$. It is regarded by the World Health Organization as one of the five major endemic infectious parasitic diseases worldwide and occurs in 98 countries $^{2}$, including 18 in the Americas. In 2013, 47,492 cases were notified in 16 American countries, of which $78.8 \%$ (37,402 cases) occurred in Brazil and countries of the Andean subregion ${ }^{3}$.

The diagnosis of ATL consists of the detection of the parasite or a test that determines its presence in the infected host. The most frequently used tests are: direct search for parasites in lesions and Montenegro skin test (MST). MST measures hypersensibility reactions to injection of Leishmania antigen. Serological tests, such as indirect immunofluorescence (IF) and enzyme immunoassay (EIA), are not commonly used since they do not distinguish between present and previous infections and may provide inconsistent results, having a cross-reaction with other diseases, such as Chagas disease $e^{4,5}$. The detection of parasites by direct search methods and MST, which, along with clinical and epidemiological aspects of the lesion form the basis of ATL diagnoses, are recommended in public health centers ${ }^{6}$.

Currently-used laboratory tests have low sensitivity and operational drawbacks in endemic areas, since they often involve slow and laborious procedures ${ }^{7,8}$. 
Polymerase chain reaction (PCR) affords high sensitivity and specificity, and it is considered a useful method for diagnosing Leishmania infection ${ }^{9}$ using clinical specimens, particularly biopsy samples. Although different molecular techniques have been evaluated for the diagnosis of LT, PCR is the main molecular technique used, as it presents advantages such as speed, sensitivity and high specificity when compared to conventional techniques based on direct research and culture ${ }^{9-12}$. Various target genes have been used for the genus or species-specific diagnosis of tegumentary leishmaniasis. However, despite having lower specificity than other genes, the most commonly used target gene is kDNA, due to the high copy number of kDNA minicircles (around 10,000 per cell). Furthermore, degenerate primers are widely used in PCR-kDNA assays, making possible to afford a reduction in specificity. Other genes such as ITS, HSP70 and glucose-6-phosphate dehydrogenase has also been used with variable sensitivity and specificity ${ }^{13-17}$.

PCR is the most commonly used method for detecting Leishmania DNA in samples taken from skin lesions. An alternative method that reduces the risk of contamination, for it decreases sample handling, is real-time PCR (qPCR). However, this method is available in a limited number of research centers and it is not routinely used in ATL diagnosis. Real-time PCR with kDNA and HSP70 genes as targets is a promising method, not only for detecting Leishmania DNA, but also for differentiating Leishmania species in samples taken from skin lesions, using High Resolution Melting $(\mathrm{HRM})^{18-20}$. Current methods used to diagnose ATL, such as parasite examinations, histopathology and molecular analysis, use samples taken by using invasive methods, mainly lesion biopsies ${ }^{21}$. Invasive sampling methods are difficult to perform in settings with limited resources and without specialized training ${ }^{22,23}$. Additionally, biopsies can often be delicate, uncomfortable and painful for the patient ${ }^{24}$, and may cause bleeding at the biopsy site. The majority of researches on the detection of Leishmania DNA focus on biopsy samples, and few studies have evaluated the use of swabs, a relatively noninvasive sampling method. Matsumoto et al. ${ }^{25}$, observed that PCR-based diagnoses of cutaneous leishmaniasis using different sampling methods (scraping, suction and biopsy) showed similar levels of sensitivity. Recently, a study of qPCR-based diagnoses performed on swab samples from patients with cutaneous leishmaniasis using $18 \mathrm{~S}$ rDNA as a target, observed an overall sensitivity of $97.5 \%$ and specificity of $84 \%{ }^{26}$. The findings have also shown that test sensitivity was greater than the one of tests performed on samples collected using aspiration and procedures that are more painful.

Noninvasive sample with swab can be collected in rural areas and tested in central laboratories, thus ensuring efficient, accurate and reliable PCR amplification. The use of noninvasive sampling, along with sensitive and simple molecular analysis, can be a valuable tool for overcoming the challenges of diagnosing tegumentary leishmaniasis. Moreover, swab samples afford greater flexibility when it comes to storage, as samples can be transported and stored at room temperature or at $4{ }^{\circ} \mathrm{C}$, or ever conserved for long periods at $-20{ }^{\circ} \mathrm{C}^{26}$.

Based on a sample of patients treated at the Leishmaniasis Clinic of the Emílio Ribas Institute of Infectious Diseases, this study aims to evaluate the efficiency of PCR-based noninvasive methods for diagnosing ATL, by comparing the sensitivity and specificity of DNA detection in biopsy samples and swab samples, as well as measuring and comparing the effectiveness of different Leishmania DNA targets in detecting leishmaniasis on swab samples collected from cutaneous and mucosal lesions.

\section{MATERIALS AND METHODS}

\section{Patients and Healthy Volunteers}

The study sample comprised 25 patients with leishmaniasis confirmed by PCR-kDNA assays performed on biopsy samples considered the gold standard test for leishmaniasis in our casuistic and who were attended at the Leishmaniasis Clinic of the Emilio Ribas Institute of Infectious Diseases of the Department of Health of the State of São Paulo (IIER, acronym in Portuguese), between December 2013 and March 2015. Included in this group were all patients referred for diagnosis of ATL in the study period and who agreed to participate in the study. The control group comprised 10 volunteers whose samples were collected from the intact nasal septum. This study was approved by the Ethics Committee of IIER $\left(\mathrm{N}^{\circ}\right.$ 07801112.1.0000.0061 and Opinion $\left.\mathrm{N}^{\circ} 326 / 644\right)$ and Research Ethics Committees of the Faculty of Medicine at University of São Paulo (Application N ${ }^{\circ}$ 069/14). All participants were informed about study procedures and signed an informed consent form.

\section{Obtaining the biopsy}

Biopsies were performed to obtain a sample of cutaneous and/or mucosal lesions in accordance with the procedures established by the clinic. Two small biopsy samples (approximately $3 \mathrm{~mm}$ in size) were obtained: one for on-site routine histopathological examination at the hospital; and one for direct search, Leishmania culture, and PCR. Biopsies from patients with LC were taken from the margin of the lesion. 


\section{Swab samples}

The samples of the cutaneous and mucosal lesions from patients were collected using a cotton swab, as described by Mimori et al. ${ }^{27}$ and Boggild et al. ${ }^{28}$, after removing any overlapping scabs or crusts with a moist gauze and cleaning the wound site and the surrounding area using ethanol $70 \%$, the swab is rubbed in a clockwise direction over the lesion surface. Samples from nasal mucosa of healthy volunteers were collect by swab and performed for detection of DNA from Leishmania.

\section{DNA extraction}

The tip of the swab was then cut with sterile scissors and immediately placed in $1.5 \mathrm{~mL}$ tubes containing $700 \mu \mathrm{L}$ of $100 \%$ ethanol, and subsequently stored at $-20^{\circ} \mathrm{C}$ prior to DNA extraction. Swab samples were initially centrifuged at $3,000 \times \mathrm{g}$ for five minutes, after which ethanol was discarded. DNA extraction was then performed using the QIAamp DNA Mini Kit (QIAGEN Inc., Germany) according to manufacturer's instructions. The samples were quantified using a NanoDrop spectrophotometer (Thermo Fisher Scientific Inc., USA) and 50 ng of DNA were used to perform PCR for each target gene. Only samples found to have a ratio of absorbance at 260 and $280 \mathrm{~nm}$ between -1.6 and -2.0 were used.

\section{Polymerase Chain Reaction with kinetoplast DNA (kDNA) minicircle}

The primers kDNA 20 (5'-GG KAG GGG CGT TCT SCG AA-3') and kDNA 22 (5'-SSS WCT ATW TTA ACA CAA CCC C-3'), described by Volpini et al..$^{29}$, were used for the conserved region of the leishmania kinetoplast DNA (kDNA), which amplified the $120 \mathrm{bp}$ fragments. The final volume of the reaction mixture was $20 \mu \mathrm{L}: 11.1 \mu \mathrm{L} \mathrm{H} \mathrm{H}_{2} \mathrm{O}$, $0.8 \mu \mathrm{L} 25 \mathrm{mM} \mathrm{MgCl}, 0.75 \mu \mathrm{L}$ of each primer $(10 \mathrm{mM})$, $0.2 \mu \mathrm{L}$ Taq Polymerase (Thermo Fisher Scientific), $2.0 \mu \mathrm{L}$ $10 \mathrm{X}$ buffer, $0.4 \mu \mathrm{L} 10 \mathrm{mM}$ dNTPs, and $4.0 \mu \mathrm{L}$ DNA sample. Amplification was performed in a Veriti ${ }^{\circledR}$ Thermal Cycler (Life Technologies, Carlsbad, California, USA). The cycling conditions comprised an initial denaturation step at $94{ }^{\circ} \mathrm{C}$ for four minutes, followed by 35 cycles at $94{ }^{\circ} \mathrm{C}$ for one minute, $58^{\circ} \mathrm{C}$ for one minute, and $72^{\circ} \mathrm{C}$ for 30 seconds, and a final extension step at $72{ }^{\circ} \mathrm{C}$ for five minutes.

Polymerase Chain Reaction to detect DNA using the target gene HSP70 (70 kDa heat shock protein)

The following leishmania-specific primers were used:
F1E (5'- CCC TCG TGT CGG ACT TCT T-3') and R1E (5'- CTC CGT CTG CTT GCT CTT G -3'), which amplified the $125 \mathrm{bp}$ fragments. The final volume of the reaction mixture was $25 \mu \mathrm{L}: 16.05 \mu \mathrm{L} \mathrm{H} 2 \mathrm{O}, 1.5 \mu \mathrm{L} 25 \mathrm{mM} \mathrm{MgCl}_{2}$, $1.5 \mu \mathrm{L}(10 \mathrm{mM})$ of each of the primers F1E and R1E, $0.2 \mu \mathrm{L}$ Taq Polymerase (Thermo Fisher Scientific), $2.5 \mu \mathrm{L}$ $10 \mathrm{X}$ buffer, $0.75 \mu \mathrm{L} 10 \mathrm{mM}$ dNTPs. and $1.0 \mu \mathrm{L}$ DNA sample. Amplification was performed in a Veriti ${ }^{\circledR}$ Thermal Cycler (Life Technologies, Carlsbad, California, USA). Cycling conditions comprised an initial denaturation step at $94^{\circ} \mathrm{C}$ for two minutes, followed by 35 cycles at $94^{\circ} \mathrm{C}$ for 30 seconds, $60{ }^{\circ} \mathrm{C}$ for 30 seconds, and $72{ }^{\circ} \mathrm{C}$ for 30 seconds, and a final extension step at $72{ }^{\circ} \mathrm{C}$ for seven minutes.

\section{Analysis of the PCR products}

PCR products were electrophoresed on $2 \%$ agarose gels at 80 volts for one hour (Biotools/M\&B Laboratories, S. A., Uniscience of Brazil, São Paulo, Brazil). After staining the gel with a $0.5 \mathrm{ug} / \mathrm{ml}$ ethidium bromide solution, amplification was visualized under ultraviolet illumination using the MacroVue ${ }^{\mathrm{TM}}$ UV transilluminator (Pharmacia Biotech, Uppsala, Sweden) and photographed using with a Canon Powershot S215 digital camera (Canon, NY, USA). A 100 bp DNA ladder molecular weight marker (Invitrogen Life Technologies, São Paulo, Brazil) was used for fragment size determination.

\section{Restriction Fragment Length Polymorphism Analysis of KDNA (PCR-RFLP)}

kDNA reaction products were digested using the restriction enzyme HaeIII FastDigest ${ }^{\circledR}$ (Themo Fisher Scientific) in accordance with the manufacturer's instructions: $10 \mu \mathrm{l}$ of positive PCR products were digested at $37^{\circ} \mathrm{C}$ for five minutes with $10 \mathrm{U}$ of the restriction enzyme, specific buffer and ultrapure water in final volume of $30 \mu$ l.

The products obtained from this procedure were electrophoresed on $10 \%$ polyacrylamide gel and stained with ethidium bromide. According to Volpini et al. ${ }^{30}$, this technique affords differentiation between $L$. (V.) braziliensis and $L$. (L.) amazonensis that may otherwise be difficult to determine using cleavage pattern in samples infected with this species.

Polymerase Chain Reaction in Real Time (qPCR) using SYBR Green

This technique was performed using leishmania-specific primers F1E (5'- CCC TCG TGT CGG ACT TCT T-3') and R1E (5'- CTC CGT CTG CTT GCT CTT G -3'). The final 
volume of the real time PCR reaction mixture was $25 \mu \mathrm{L}$ : $10.5 \mu \mathrm{L} \mathrm{H}_{2} \mathrm{O}, 12.5 \mu \mathrm{L}$ Mix (UBS), $0.5 \mu \mathrm{L}$ (10 mM) of each of the primers, and $1.0 \mu \mathrm{L}$ sample in $30 \mathrm{ng} / \mu \mathrm{L}$ concentration of DNA. Samples were analyzed in triplicate.

\section{Reference Standards}

The reference standard was considered the result of the PCR-kDNA on biopsy samples, in comparison with results of tests conducted in the present study. The diagnosis of tegumentary leishmaniasis was confirmed when the above test yielded a positive result.

\section{Statistical Analysis}

Statistical analyses were performed using Fisher's exact test and chi-square test using Epi Info ${ }^{\circledR}$ 7.2. The level of significance was set at $\mathrm{p} \leq 0.05$.

\section{RESULTS}

The study sample comprised 25 patients, 14 of whom were suspected of having mucosal leishmaniasis (ML), and 11 suspected of having cutaneous leishmaniasis (CL). Four of the patients suspected of having mucosal leishmaniasis $(28.6 \%)$ had a skin lesions history. A total of 27 samples were collected from these patients: 15 samples from patients suspected of having ML, and 12 from patients suspected of having CL. The total number of samples was greater than the total number of patients, because one of the patients suspected of having ML had nasal and oral mucosa lesions, and one of the patients suspected of having CL had more than one lesion. All patients tested positive by PCR-kDNA performed on biopsy samples and were thus diagnosed as having ATL.

After confirming leishmaniasis, PCR-kDNA was performed on swab samples to determine the sensitivity of the diagnosis, yielded positive results in 25 samples and negative results in two samples. The sensitivity and specificity of the PCR assay was $92.6 \%$ and $80 \%$, respectively, since there two samples from negative control patients (healthy volunteers) had positive results.

A comparison between swab samples and biopsy samples showed an agreement rate of $86.2 \%$ : PCR-kDNA testing yielded positives results in all 27 biopsy samples and 25 swab samples ( $\mathrm{p}=0.11$ ), as shown in Table 1 .

A separate analysis between ML and CL patients demonstrated positivity rates of $93.3 \%$ (14/15) and $91.7 \%$ $(11 / 12)$, respectively. However, this difference was not statistically significant $(\mathrm{p}=0.72)$. The analysis of HaeIII enzyme cleavage patterns of the 25 swab samples confirmed the presence of L. braziliensis (80 and $40 \mathrm{bp}$ ).
Table 1 - Results of the diagnostic tests of 27 samples from patients suspected of having American Tegumentary Leishmaniasis treated at the IIER, between December 2013 and March 2015

\begin{tabular}{lccc}
\hline Biopsy & \multicolumn{3}{c}{ Swab samples } \\
\cline { 2 - 4 } samples & Positive & Negative & Total \\
\hline Positive & 25 & 2 & 27 \\
Negative & 0 & 0 & 0 \\
\hline Total & 25 & 2 & 27 \\
\hline
\end{tabular}

PCR assays using HSP70 primers performed on 22 of 25 biopsy samples yielded a positivity rate of $81.82 \%$, or 18 samples: 10 samples from patients suspected of having ML, and eight from patients suspected of having CL. All 22 biopsy samples were PCR-kDNA positive.

PCR assays using HSP70 primers performed on the 27 swab samples yielded 11 positive and 16 negative results. The sensitivity and specificity of this PCR assay was $40.7 \%$ and $100 \%$, respectively. All samples from healthy volunteers were negative for the target gene HSP 70. A separate analysis between ML and CL patients demonstrated positivity rates of $26.6 \%(4 / 15)$ and $58.3 \%$ $(7 / 12)$, respectively $(\mathrm{p}=0.12)$.

One swab sample from a patient suspected of having $\mathrm{CL}$ tested negative with the PCR-kDNA assay and positive with the PCR-HSP70 assay. Fifteen of the 16 swab samples that tested negative in the PCR-HSP70 assay were PCR-kDNA positive and one was negative on both tests $(\mathrm{p}=0.66)$.

Regarding the PCR-HSP70, differences were found in 13 of the 22 samples (two of the biopsy samples tested negative and 11 tested positive) as shown in Table 2. In one patient suspected of having of ML and one suspected of having CL, the biopsy samples tested negative and the swab samples tested positive. Eleven patients tested positive in biopsy samples and negative in swab samples. However, when the biopsy samples collected from these patients were tested using PCR-kDNA, all tested positive: eight ML patients, and three CL patients. The differences found

Table 2 - Results of diagnostic tests with HSP70 primers performed on 22 biopsy samples and 22 swab samples from patients suspected of having American Tegumentary Leishmaniasis treated at the IIER, between December 2013 and March 2015

\begin{tabular}{lccc}
\hline PCR-HSP70 & \multicolumn{3}{c}{ PCR-HSP70 Swab } \\
\cline { 2 - 4 } Biopsy & Positive & Negative & Total \\
\hline Positive & 7 & 11 & 18 \\
Negative & 2 & 2 & 4 \\
\hline Total & 9 & 13 & 22 \\
\hline
\end{tabular}


between biopsy samples and swab samples using HSP70 primers were not statistically significant $(\mathrm{p}=0.55)$.

Regarding qPCR assays using HSP70 primers, 22 biopsy samples yielded 12 positive and 10 negative results. All positive samples were consistent with the results of conventional PCR-HSP70 and PCR-kDNA also analyzing biopsy samples. However, six of the 10 negative samples, tested positive - by conventional PCR-HSP70 and PCRkDNA; three of which were from ML patients, and three from CL patients. The analysis shows that the conventional PCR-HSP70 is more efficient than qPCR-HSP70 when using biopsy samples $(\mathrm{p}=0.02)$.

Quantitative qPCR assays performed on 27 swab samples yielded nine positive results and 18 negative results. The sensitivity of qPCR was $33.3 \%$. Five of the nine positive samples (55.6\%) tested negative using conventional PCR assay for the same target (Table 3), two of which were from CL patients and three from ML patients; while seven of the 18 negative samples, tested positive using conventional PCR assay, four of which were from CL patients, and three from ML patients.

Table 3 - Results of the conventional PCR and qPCR- with the same target performed on 27 swab samples from patients suspected of having American Tegumentary Leishmaniasis treated at the IIER, between December 2013 and March 2015

\begin{tabular}{lccc}
\hline PCR-HSP70 & \multicolumn{3}{c}{ qPCR-HSP70 } \\
\cline { 2 - 4 } & Positive & Negative & Total \\
\hline Positive & 4 & 7 & 11 \\
Negative & 5 & 11 & 16 \\
\hline Total & 9 & 18 & 27 \\
\hline
\end{tabular}

A comparison of the results obtained from swab samples and biopsy samples using qPCR assays found a quantitative difference. However, this difference was not statistically significant $(\mathrm{p}=0.45)$. From 12 patients that tested positive using qPCR performed on biopsy samples, only five tested positive using assays performed on swab samples, three of which were ML patients and two were CL patients. In line with these findings, only seven of the 10 patients that tested negative using qPCR performed on biopsy samples, tested negative using assays performed on swab samples, of which five were ML patients and two were CL patients. Therefore, qPCR testing performed on biopsy and swab samples yielded different results in 10 of the 22 patients.

A separate analysis between ML and CL patients demonstrated positivity rates of $26.7 \%$ (4/15) and $41.7 \%$ (5/12) respectively, showing that the effectiveness of the test was the same regardless of the form of leishmaniasis $(\mathrm{p}=0.44)$.
The following levels of agreement were observed between the molecular tests: swab samples taken from ML patients $44 \%$ between PCR-kDNA and PCR-HSP70 $(\mathrm{p}=0.73), 40 \%$ between PCR-kDNA and $\mathrm{qPCR}(\mathrm{p}=0.73)$, and $72 \%$ between PCR-HSP70 and qPCR $(\mathrm{p}=0.75)$; swab samples taken from CL patients 53,8\% between PCR-kDNA and PCR-HSP70 $(\mathrm{p}=0.5), 33,3 \%$ between PCR-kDNA and $q$ PCR $(p=0.67)$, and $50 \%$ between PCR-HSP70 and qPCR $(p=0.69)$.

\section{DISCUSSION}

The increased availability of molecular methods with high sensitivity and specificity, together with the development of less invasive sample collection methods, have opened the path to more efficient diagnosis and a greater understanding of infection with Leishmania spp. As such, molecular methods have emerged as the new "gold standard" for the diagnosis of cutaneous leishmaniasis ${ }^{7}$, and are particularly useful for forms of the disease that have low parasite loads, such as mucosal leishmaniasis ${ }^{30}$.

The present study assessed the efficiency of non-invasive diagnostic method - the use of a swab - for detecting Leishmania using PCR performed with kDNA and HSP70 targets and qPCR to detect HSP70. The performance of tests on swab samples was compatible with that of PCR performed on biopsy samples for tegumentary leishmaniasis analyzed in this study. These results corroborate the findings of Boggild et al. ${ }^{28}$ and Ferreira et al. ${ }^{31}$, who analyzed the use of swabs for sample collection on human patients and dogs, respectively, suspected of having ML, and those of Mimori et al. $^{27}$, who observed sensitivity of $93.8 \%$ in detection of five species of Leishmania spp. using PCR performed on swab samples from CL patients.

The main problem faced by this study was the low level of DNA recovery from the swabs; however, this sampling method proved to be particularly useful, since it is painless, easy to perform without the need for specially qualified personnel, and it can be performed in any setting. Unlike invasive methods, such as skin biopsies, swab sampling minimizes the risk of iatrogenic infections that may compromise diagnosis and patient recovery ${ }^{32}$.

The findings of this study show that the high efficiency of PCR performed with kDNA primers in the detection of parasites in swab samples affords the opportunity of noninvasive access to tissues, and it is a promising alternative to conventional methods both for ML and CL patients. Similar results were found by Figueroa et al..$^{23}$ in ML patients, reinforcing the findings of the present study with respect to the good performance of this test. 
The use of kDNA as an amplification target in swab samples demonstrated favorable results due to its abundance on Leishmania DNA spp., which, according to Mouttaki et al. ${ }^{33}$ is around 10,000 copies per parasite. However, false-positive results, due to extremely sensitive reactions, are a concern ${ }^{29}$. This study used degenerate primers that paired Leishmania kDNA sequences, which explains the low specificity achieved.

False-positive results were observed in the application of HSP70 primers to swab samples, which confirms its low sensitivity. The erroneous results were confirmed by routine and molecular tests using other primers and biopsy results. A difference in the efficiency of this test was also found between ML and CL patients, with better results in CL patients. This finding could be explained by the fact that larger quantities of parasites are found in skin lesions than in mucosal lesions. Despite the low rates found in this study, protocols using alterations in nucleotide bases of HSP70 coding sequences and different melting times, enable differentiation between species of Leishmania in molecular tests performed on biopsy samples ${ }^{20}$, which affords a rapid and effective treatment for the disease.

PCR-kDNA was more efficient than the HSP70 ones which showed low sensitivity in swab samples. These findings confirm that primers targeting the parasite kinetoplast DNA, are more efficient than the ones from HSP70 gene sequence in swab samples. Once again, these findings are justified by the abundance, specificity and repetitive nature of $\mathrm{kDNA}^{18}$, which avoid false-negative results. The findings also show that the sensitivity of PCR using HSP70 primers was higher in biopsy samples than in swab samples, mainly in those from CL patients. This is explained by the low level of DNA recovery from swabs and the fact that this is a single copy gene of Leishmania ${ }^{34}$ and it is more prevalent in biopsy samples.

The efficiency of qPCR using HSP70 primers was low in both swab samples and biopsy samples, but it was higher in the later one. The results show that conventional PCR was more efficient in the case of swab samples. These results diverge from the findings of a study conducted by Adams et $a l .{ }^{26}$ of qPCR performed using 18S rDNA primers on swab samples collected from CL patients, which observed sensitivity and specificity of $98 \%$ and $84 \%$, respectively, showing that this test had good applicability and accuracy.

No statistically significant difference was found between the three tests with respect to the different forms of the disease. However, it is evident that for swab samples, PCR-kDNA test is more efficient than PCR-HSP70 and qPCR tests, while for biopsy samples, PCR-kDNA and PCR-HSP70 tests are more efficient than qPCR test.

The findings of this study show that skin and mucosal samples collected using a swab have a significant diagnostic potential for detection of ATL using PCR due to this test high positivity rates, which are equivalent to those obtained from biopsy samples using the same primers. The use of swabs has various applications in the molecular diagnosis of human and canine leishmaniasis ${ }^{31}$.

Although the present study shows that noninvasive sampling for the diagnosis of leishmaniasis using PCR is satisfactory, further research is required using other primers. The findings also show that PCR-kDNA testing is more efficient than qPCR testing with HSP70 primers. Swab sampling affords numerous advantages over biopsy samples, such as lower costs, patient comfort and security, and ease of performance, which in turn reduces the need for highly qualified personnel for sample collection.

\section{REFERENCES}

1. Jhingran A, Chatterjee M, Madhubala R. Leishmaniasis: epidemiological trends and diagnosis. In: Myler PJ, Fasel $\mathrm{N}$, editors. Leishmania after the genome. Norfolk: Caister Academic Press; 2008. p.1-14.

2. World Health Organization. Leishmaniasis: situation and trends. [cited 2016 Jan 07]. Available from: http://www.who.int/gho/ neglected_diseases/leishmaniasis/en/

3. Pan American Health Organization. Leishmaniases: epidemiological report of the Americas. Rep Leishmaniases. 2015;3:1-4.

4. Faber WR, Oskam L, van Gool T, Kroon NC, Knegt-Junk $\mathrm{KJ}$, Hofwegen $\mathrm{H}$, et al. Value of diagnostic techniques for cutaneous leishmaniasis. J Am Acad Dermatol. 2003;49:70-4.

5. Venazzi EA, Roberto AC, Barbosa-Tessmann IP, Zanzarini PD, Lonardoni MV, Silveira TG. Detection of Leishmania (Viannia) DNA in blood from patients with American cutaneous leishmaniasis. Exp Parasitol. 2007;115:399-402.

6. Goto H, Lindoso JA. Current diagnosis and treatment of cutaneous and mucocutaneous leishmaniasis. Expert Rev Anti Infect Ther. 2010;8: 419-33.

7. Weigle KA, Labrada LA, Lozano C, Santrich C, Barker DC. PCR-based diagnosis of acute and chronic cutaneous leishmaniasis caused by Leishmania (Viannia). J Clin Microbiol. 2002;40:601-6.

8. Safaei A, Motazedian MH, Vasei M. Polymerase chain reaction for diagnosis of cutaneous leishmaniasis in histologically positive, suspicious and negative skin biopsies. Dermatology. 2002;205:18-24.

9. Reithinger R, Dujardin JC. Molecular diagnosis of leishmaniasis: current status and future applications. J Clin Microbiol. 2007;45:21-5.

10. Belli A, Rodriguez B, Aviles H, Harris E. Simplified polymerase chain reaction detection of new world Leishmania in clinical specimens of cutaneous leishmaniasis. Am J Trop Med Hyg. 1998;58:102-9. 
11. Satow MM, Yamashiro-Kanashiro EH, Rocha MC, Oyafuso LK, Soler RC, Cotrim PC, et al. Applicability of kDNA-PCR for routine diagnosis of American tegumentary leishmaniasis in a tertiary reference hospital. Rev Inst Med Trop Sao Paulo. 2013;55:393-9.

12. Singh $\mathrm{S}$. New developments in diagnosis of leishmaniasis. Indian J Med Res. 2006;123:311-30.

13. Uliana SR, Nelson K, Beverley SM, Camargo EP, Floeter-Winter LM. Discrimination amongst Leishmania by polymerase chain reaction and hybridization with small subunit ribosomal DNA derived oligonucleotides. J Eukaryot Microbiol. 1994;41:324-30.

14. Uliana SR, Affonso MH, Camargo EP, Floeter-Winter LM. Leishmania: genus identification based on a specific sequence of the 18 S ribosomal RNA sequence. Exp Parasitol. 1991;72:157-63.

15. Castilho TM, Shaw JJ, Floeter-Winter LM. New PCR assay using glucose-6-phosphate dehydrogenase for identification of Leishmania species. J Clin Microbiol. 2003;41:540-6.

16. Fraga J, Montalvo AM, De Doncker S, Dujardin JC, Van der Auwera G. Phylogeny of Leishmania species based on the heat-shock protein 70 gene. Infect Genet Evol. 2010;10:238-45.

17. Montalvo AM, Fraga J, Monzote L, Montano I, De Doncker S, Dujardin JC, et al. Heat-shock protein 70 PCR-RFLP: a universal simple tool for Leishmania species discrimination in the New and Old World. Parasitology. 2010;137:1159-68.

18. Weirather JL, Jeronimo SM, Gautam S, Sundar S, Kang M, Kurtz MA, et al. Serial quantitative PCR assay for detection, species discrimination, and quantification of Leishmania spp. in human samples. J Clin Microbiol. 2011;49:3892-904.

19. Jara M, Adaui V, Valencia BM, Martinez D, Alba M, Castrillon C, et al. Real-time PCR assay for detection and quantification of Leishmania (Viannia) organisms in skin and mucosal lesions: exploratory study of parasite load and clinical parameters. J Clin Microbiol. 2013;51:1826-33.

20. Zampieri RA, Laranjeira-Silva MF, Muxel SM, Stocco de Lima AC, Shaw JJ, Floeter-Winter LM. High resolution melting analysis targeting hsp70 as a fast and efficient method for the discrimination of Leishmania species. PLoS Negl Trop Dis. 2016;10:e0004485.

21 David CV, Craft N. Cutaneous and mucocutaneous leishmaniasis. Dermatol Ther. 2009;22:491-502.

22. Boggild AK, Valencia BM, Espinosa D, Veland N, Ramos AP, Arevalo J, et al. Detection and species identification of Leishmania DNA from filter paper lesion impressions for patients with American cutaneous leishmaniasis. Clin Infect Dis. 2010;50:e1-6.

23. Figueroa RA, Lozano LE, Romero IC, Cardona MT, Prager M, Pacheco R, et al. Detection of Leishmania in unaffected mucosal tissues of patients with cutaneous leishmaniasis caused by Leishmania (Viannia) species. J Infect Dis. 2009;200:638-46.
24. Martins L, Alexandrino A, Guimarães G. Detection of Leishmania braziliensis DNA in American tegumentary leishmaniasis patients. Rev Saude Publica. 2010;44:571-4.

25. Matsumoto T, Hashiguchi Y, Gomez EA, Calvopiña MH, Nonaka $\mathrm{S}$, Saya $\mathrm{H}$, et al. Comparison of PCR results using scrape/ exudate, syringe-sucked fluid and biopsy samples for diagnosis of cutaneous leishmaniasis in Ecuador. Trans R Soc Trop Med Hyg. 1999;93:606-7.

26. Adams ER, Gomez MA, Scheske L, Rios R, Marquez R, Cossio A, et al. Sensitive diagnosis of cutaneous leishmaniasis by lesion swab sampling coupled to qPCR. Parasitology. 2014;141:18917.

27. Mimori T, Matsumoto T, Calvopiña MH, Gomez EA, Saya H, Katakura K, et al. Usefulness of sampling with cotton swab for PCR-diagnosis of cutaneous leishmaniasis in the New World. Acta Trop. 2002;81:197-202.

28. Boggild AK, Valencia BM, Veland N, Pilar Ramos A, Calderon F, Arevalo J, et al. Non-invasive cytology brush PCR diagnostic testing in mucosal leishmaniasis: superior performance to conventional biopsy with histopathology. PLoS One. 2011;6:e26395.

29. Volpini AC, Passos VM, Oliveira GC, Romanha AJ. PCR-RFLP to identify Leishmania (Viannia) braziliensis and L. (Leishmania) amazonensis causing American cutaneous leishmaniasis. Acta Trop. 2004;90:31-7.

30. Ovalle Bracho C, Porras de Quintana L, Muvdi Arenas S, Rios Parra M. Polymerase chain reaction with two molecular targets in mucosal leishmaniasis' diagnosis: a validation study. Mem Inst Oswaldo Cruz. 2007;102:549-54.

31. Ferreira SA, Almeida GG, Silva SO, Vogas GP, Fujiwara RT, de Andrade AS, et al. Nasal, oral and ear swabs for canine visceral leishmaniasis diagnosis: new practical approaches for detection of Leishmania infantum DNA. PLoS Negl Trop Dis. 2013;7:e2150.

32. Rodrigues EH, Felinto de Brito ME, Mendonça MG, Werkhäuser RP, Coutinho EM, Souza WV, et al. Evaluation of PCR for diagnosis of American cutaneous leishmaniasis in an area of endemicity in northeastern Brazil. J Clin Microbiol. 2002;40:3572-6.

33. Mouttaki T, Morales-Yuste M, Merino-Espinosa G, Chiheb S, Fellah H, Martin-Sanchez J, et al. Molecular diagnosis of cutaneous leishmaniasis and identification of the causative Leishmania species in Morocco by using three PCR-based assays. Parasit Vectors. 2014;7:420.

34. Folgueira C, Quijada L, Soto M, Abanades DR, Alonso C, Requena JM. The translational efficiencies of the two Leishmania infantum HSP70 mRNAs, differing in their 3'-untranslated regions, are affected by shifts in the temperature of growth through different mechanisms. J Biol Chem. 2005;280:3517283. 